\author{
G. Blažienè \\ (Institute of the Lithuanian Language, Vilnius, Lithuania)

\section{WAS MAN NICHT ALLES IN DEN FOLIANTEN DES DEUTSCHEN ORDENS FINDET}

\title{
G. Blažienè. The Many Discoveries in the Folios of the Teutonic Order
}

The present article can be considered a continuation of the article $« \mathrm{Zu}$ den russischen Personenamen in den Folianten des Deutschen Ordens» (On Russian Personal Names in the Folios of the Teutonic Order), which appeared in 2018 in this journal. That article examined the ethnic composition of densely populated Sambia. It was shown that it is impossible to postulate a homogenous population for the region. The naming of inhabitants and their personal names unambiguously demonstrate beyond doubt that they belonged to different ethnic groups. Sambia was inhabited by Prussians, Germans, Lithuanians and even Russians. In other Prussian lands Polish personal names can also be found. The present article focuses on Lithuanians who settled in the villages of Rogehnen and Schorschehnen of the Wargen district in 1372; a study of their names suggests that the last names might have originated from nicknames. Lithuanian personal names reveal both the ethnic identity of the name-bearers and the tendency of populations to mix. It would be a huge success for research of historical, namely Prussian, proper names to discover traces of language change and to determine what language was spoken by Lithuanian and Russian newcomers. It is natural to ask oneself how fluent were they in their respective mother tongue, as well as whether they spoke Prussian or were already Germanized. These questions require a well-founded and reasoned answer. The title of the article itself - «The Many Discoveries in the Folios of the Teutonic Order» - points to the unresolved enigmas of historical demography, which could be solved by carefully assembling reliable archival data and exploring the links between empiricism and theory.

Folio 107 from the funds of the Historical Königsberg Archive kept in the Secret State Archives Prussian Cultural Heritage Foundation in Berlin has already been thoroughly studied by the author; unfortunately, some important points were missed. Since 2001, the author has been studying the Prussian appellatives that were considered place names and accidentally found another damerau in OF 107, which had previously been considered a place name. It will be briefly discussed in the article.

A fascinating drawing referring to the Latin privilege on the same page was found in the folio. It will also be mentioned in the article, as such expressive drawings are uncommon for the Order's folios.

Keywords: folios of the Teutonic Order, naming of Sambian inhabitants, personal names, ethnic diversity, Sambian multilingualism, place name and appellative, drawings in manuscripts. 


\section{Г. Блажене \\ (Институт литовского языка, Вильнюс, Литва)}

\section{И чего только не обнаружишь в фолиантах Немецкого ордена}

Данную работу можно трактовать как продолжение статьи 2018 года « $\mathrm{Zu}$ den russischen Personennamen in den Folianten des Deutschen Ordens» («O русских антропонимах в фолиантах Тевтонского ордена»), изданной в XXII томе данного журнала. В этой публикации была предпринята попытка исследовать этнический состав густонаселенной Самбии. Автором сделан вывод, что с полной уверенностью говорить о гомогенности состава жителей не приходится. Встречаемые названия жителей, их личные имена недвусмысленно указывают на их принадлежность к разным этносам. Среди жителей Самбии упоминаются пруссы, немцы, литовцы и даже русские. В других прусских землях встречается перечисление польских личных имен.

В данной статье внимание сосредоточено на литовцах, поселившихся в 1372 году в деревнях Рогенен (Rogehnen) и Шоршенен (Schorschehnen) волости Варген. Автор склоняется к выводу, что литовские фамилии могли происходить и от прозвищ. Литовские личные имена открывают национальную принадлежность их владельцев и тенденцию жителей к смешению. Для исследователей исторического словника, в данном случае, прусского, было бы большим событием и удачей установить следы мены языков и определить, на каком языке говорили литовские и русские переселенцы. Возникает вопрос, говорили ли они на своих родных языках, владели прусским или были уже онемечены. На данные вопросы нужен очень обоснованный и аргументированный ответ. Уже в названии статьи «О русских антропонимах в фолиантах Тевтонского ордена» подчеркнута неразрешенность исторической демографической загадки, однако тщательно собранные достоверные архивные материалы, а также эмпирические и теоретические взаимосвязи позволяют решить эту непростую задачу.

Автором статьи был тщательно изучен фолиант Ордена № 107 из фондов Исторического архива Кенигсберга, хранящихся в Тайном государственном архиве «Прусское культурное наследие» в Берлине, но некоторые факты остались незамеченными. С 2001 года автор исследует относсящиеся к топонимам прусские апеллятивы и обнаружила в OF 107 еще один случай употребления damerau, но не в качестве топонима, а как апеллятив. Он кратко рассмотрен в статье.

В фолианте был обнаружен рисунок, относящийся к латинской привилегии, записанной на этой странице. Таких рисунков (maniculae) в фолиантах Ордена совсем немного.

Ключевые слова: фолианты Тевтонского ордена и рисунки в документах, ономастика области Самбия, этнический состав Самбии и многоязычие; топонимы и апеллятивы. 
Diesen Beitrag kann man als Fortsetzung des im Jahre 2018 in nicht nur von mir geschätzten Reihe Beiträge zum Gedächtnis von Professor Joseph M. Tronskij veröffentlichten Beitrages «Zu den russischen Personennamen in den Folianten des Deutschen Ordens» beurteilen, wo der Versuch unternommen wurde, die ethnische Zusammensetzung im dicht besiedelten Samland näher zu ergründen und zu zeigen, dass man von der Einheitlichkeit der Bevölkerung nicht mit Sicherheit sprechen kann. Die Personennamen der Einwohner, verzeichnet im OF 107, sowie der Kontext der Urkunden zeigen prußische, deutsche, litauische und sogar russische Volkszugehörigkeit an. In anderen prußischen Ländereien ist eine polnische Personennamenschicht deutlich erkennbar. Darüber wird die Rede in den erwähnten Beiträge XXIV sein. Die Aufmerksamkeit gilt diesmal den litauischen $\mathrm{PN}$, die die nationale Zugehörigkeit der Namenträger widerspiegeln und die Tendenz der Bevölkerungsmischung offenbaren. Für die Namenforscher des historischen - in diesem Fall in erster Linie des prußischen Namengutes wäre ein großes Ereignis, die Spuren des Sprachwechsels zu entdecken und festzustellen, in welcher Sprache sich die litauischen bzw. die russichen Umsiedler unterhielten haben. Waren sie ihrer Muttersprachen kundig, kannten sie prußisch, waren sie eingedeutscht? Das sind die Fragen, die einer fundierten Antwort wert sind. Der Titel des Beitrages «Was man nicht alles in den Folianten des Deutschen Ordens findet» verbirgt auch ungelöste Rätsel der historischen Demographie, die nur dank einer sorgfältig erarbeiten Materialgrundlage sowie der Verknüpfung von Empirie und Theorie gelöst werden könnte (s. Wenzel 2009: 67ff.).

Um die nicht prußischen Personennamen des Samlandes näher zu erforschen, wurde das Namenmaterial des Kammeramtes Wargen aufgearbeitet. Diesmal haben wir die in diesem Amt überlieferten Namen der Litauer studiert. Wie bekannt soll die Behandlung der Personennamen auch mit genauer Orts- und Quellenangabe erfolgen. Nach Abschluss der Materialsammlung, nach Abwägen von Pro- und Contra-Argumenten wurde beschlossen Interpretationsmöglichkeiten der unten angeführten litauischen PN in Erwägung zu ziehen. Des Weiteren werden die Abschriften der Handfesten des OF 107 (zum OF 107 s. Blažienè 2018: 110) vorgelegt nebst den Originalen, die aussagefähig sind:

1372 [Überschrift der Urkunde] Ibidem Hancke Wapse

Wir Bruder Winrich von Knyprode homeist[er] der Brud[er] deß orden $\beta$

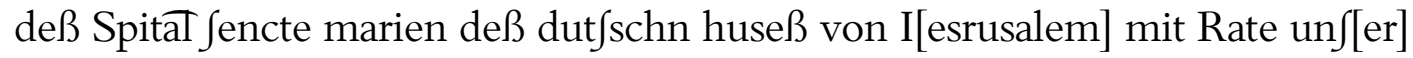


mitgebitege[r] vorlyen vnd geben Tridden dem littowe von poffiln vnd synen rechten erbn vnd nachk[omelingen] |iiij hock[en] vff dem velde c3̧u Ro gyn gelegen bynòe den greniczen alß $\int \mathrm{y}$ Im bewi $\int$ et syn von vn $\int[\mathrm{r}] \mathrm{n}$ brude[r]n mit acker we $\int e n$ welden weyden vnd alle deme daß dor c3u gehoret ffry von c3enden vnd geburlichir erbeit Erplich vnd

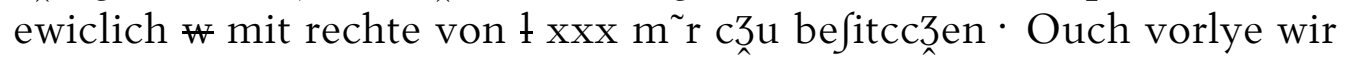
en von $\int$ undirlich[er] gnaden ap dy land eineß Iare $ß$ betwunge[n] $\mathrm{w}^{\mathrm{r}}$

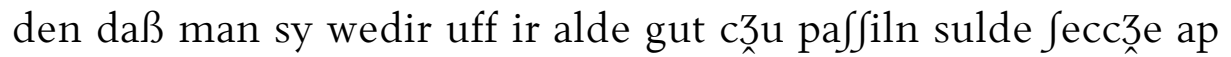
$\int \mathrm{y}$ i $ß$ begerten - fry von $\int$ ullen $\int \mathrm{y}$ vn $ß$ dynen mit pferden vnd mit mit pferden vnd mit wopen noch de $\beta$ land[eß] gewoòh[heit] $\cdot \mathrm{C} 3 \mathrm{x} u$ alle herfer

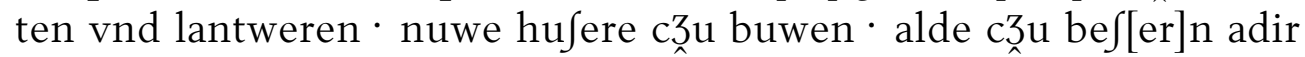
c33u brechen ' wenne $\cdot$ widick[...] vnd wo hin $\int y$ gehei $\iint e n$ we[r]den von vnß adir von vn $\int\left[\right.$ er]n bruden $\cdot$ C3u ewig[en] gedechtni $\iint \operatorname{de} \int[\mathrm{er}] \operatorname{ding}[\mathrm{e}]$ habe[n]

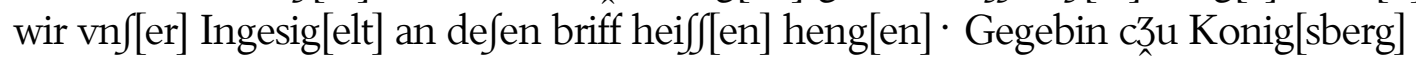
In vn $\int[\mathrm{er}] \mathrm{n} \mathrm{h}[\mathrm{e}]$ ren Jaren $\mathrm{m}^{\circ} \mathrm{ccc}^{\mathrm{o}}$ lxxij Am doòrstag noch nati ${ }^{\mathrm{t}} \mathrm{ma}[\mathrm{r}] \mathrm{ie}$ Ge3̄ūg synt vnf[er] lyben brudere Brud[er] Wulf von Beldirfh B Grof[er] Bruder Ruth[iger] von Elner obirft[er] marsch[all] Brud[er] Ulrich von frick Obirft[er] Spit[alif] vnd B[...] c3um Elbing • Bruder Swedir von pelland Tre $\int[$ ler $] \cdot$ her Nicloß vnf[er] Caplan · Reiòh ETn vnd Kune ẁo libe[n]st[...] vnfer Comp[an] vnd ander Erfam[e] lut[e]

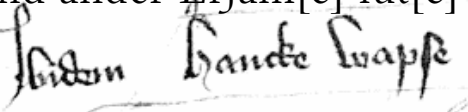

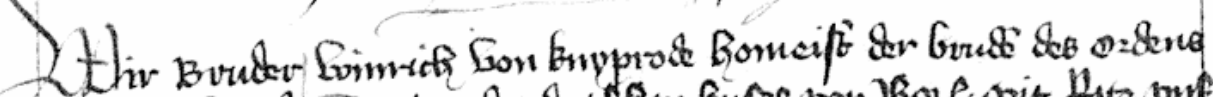

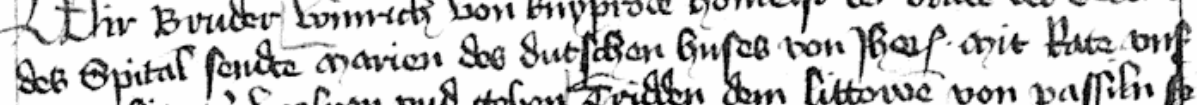

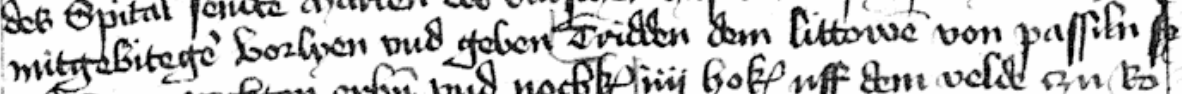

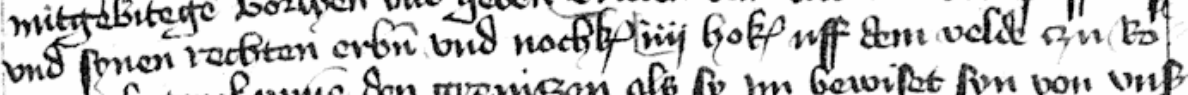

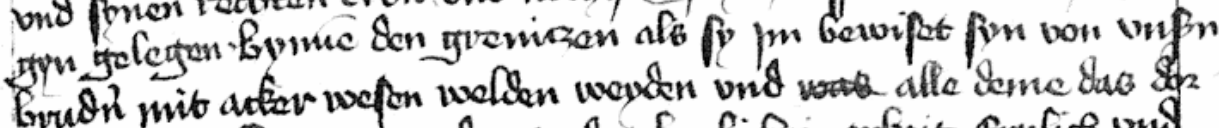

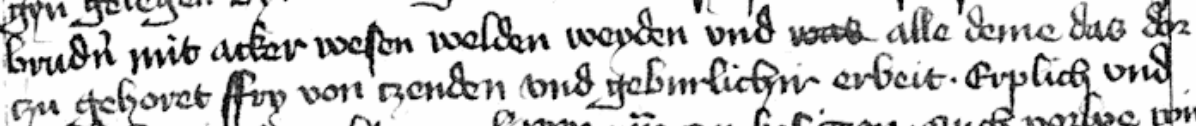

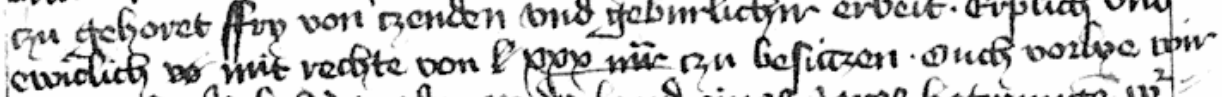

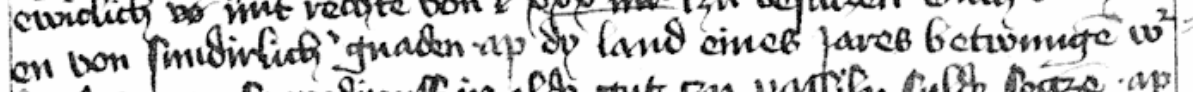

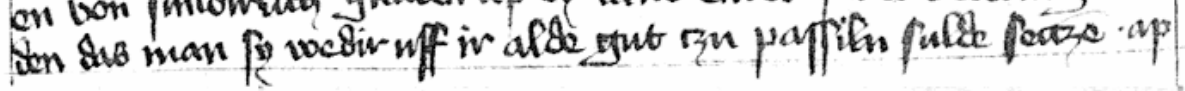

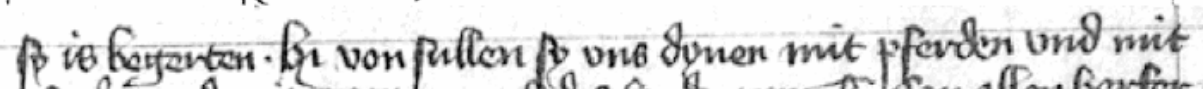

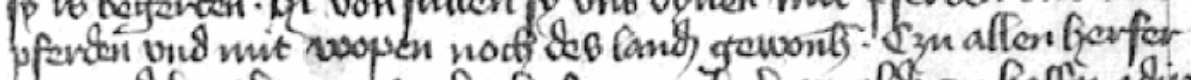

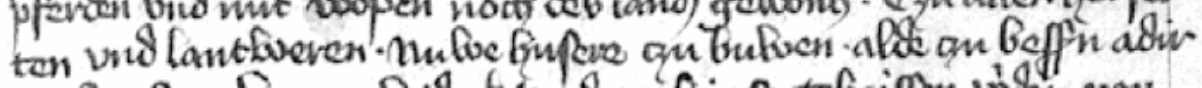

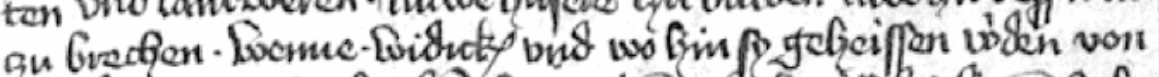

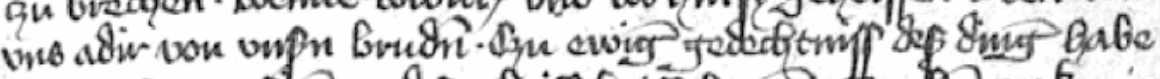

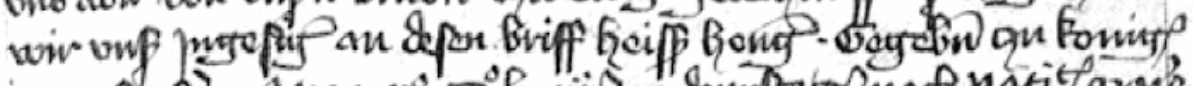

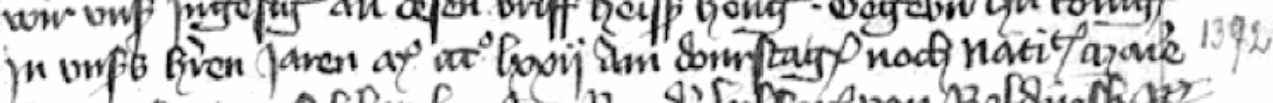

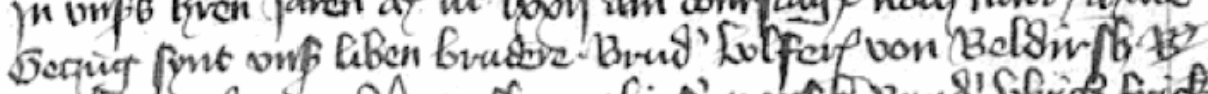

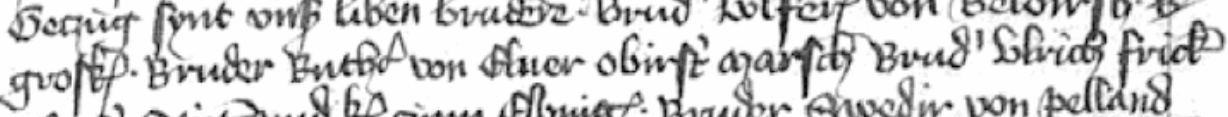

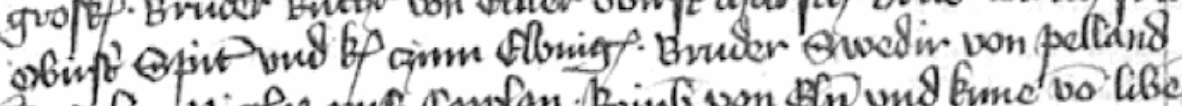

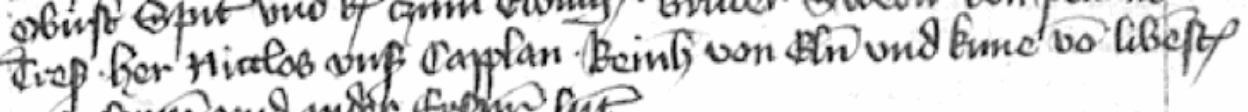

unf comp cond ander evpors out

GStA PK, XX, HA, OF, Nr. 107, Bl. 127v-128r 
In der Handfeste wird der Name des Litauers Tridden von Passiln verzeichnet. Der Begünstigte wurde mit 4 Haken des Landes (nach heutigem Maß mit 44 Hektar) im Dorf Rogyn, späteres Rogehnen, im Kirchspiel Wargen, KA Wargen (zu dem Siedlungsnamen s. Blažienė 2000: 133) beliehen. Sein Geburtsort oder Wohnort in Litauen wird auch angegeben. Möglicherweise trifft der Siedlungsname auf den im Jahre $1563 \mathrm{im}$ Amt Tilsit verzeichneten Dorfnamen Paschill zu (Diehlmann 2006: 274). Ungeachtet m. E. der unkomplizierten Deutung des Siedlungsnamens als Bildung von lit. pašile é 'Ort an einem Wald, einer Heide' oder Ableitung des lit. Präfixes $p a$-'unter, hinter' von lit. šilas 'Wald, Forst, Heide, Wildnis' (LKŽe) und trotz der Verbreitung der Heiden sowohl in Litauen als auch im ehemaligen Kleinlitauen/Preußisch-Litauen, kommt dieser Siedlungsname - Pãšilè, Pašilẽé, Pašiliaĩ (ATSŽ 230231) oder Pašilè, Pašilis (Pèteraitis 1997: 289) - nicht oft vor, besonders selten in Kleinlitauen/Preußisch-Litauen. Diese Schlussfolgerung läßt die Vermutung zu, dass der Litauer Tridden aus dem ehemaligen Kleinlitauen/Preußisch-Litauen nach Samland gekommen war und dort beliehen wurde. Zu dem Personennamen vgl. den früh belegten lit. PN 1540 Janell Traydys, des Michel Joksch instmann 3cw Tauerlakenn (Ostpr. Fol. 911 a XIX 1 187r), jetzt Taurâlaukis bei Klaipèda (Pèteraitis 1997: 402-403). Dass der Vokalwechsel in den angegebenen PN möglich war, bezeugen lit. Appellativa, vgl. lit. traidà, s. trydà, vgl. lit. tridà 'Durchfall, Diarrhöe' (LKŽe; vgl. Fraenkel 1965: 1122). Semantisch ist dieser Wechsel auch annehmbar. Die Wurzel traid- ist in der lit. Anthroponymie seit dem 13. Jh. bekannt. Der Name des Großfürsten von Litauen Traidẽnis, der von 1269 oder 1270 bis zu seinem Tode im Jahre 1282 regierte (Gudavičius, Matulevičius 2013: 64), wurde von Kazimieras Būga (1911/1958: 239) im Jahre 1911 besprochen. Būga stellt fest, dass die Wurzel Traid- nur in den Siedlungsnamen Traidžiai bei Kretinga, unweit also von dem Wohnort der im Jahre 1540 belegten Traydys, und Traidžiūnai (jetzt Traidžiū́nai s. ATSŽ 318), vgl. den lit. PN eingeordnet in die historische Kartei der Personnennamen (IAK), aufbewahrt im Institut für litauische Sprache, 1678-1693 Anna Trejdziunowa (Treidziuny) im Rayon Utena, weit von dem Belegort des uns interessierenden Personennamens entfernt (IAK), zu finden ist. Traid- wird zu lit. traid-alióti 'sich heftig gebärden; viel schwatzen' gestellt (LVKŽ 350). Es könnte die Frage entstehen, warum dieser Vorstoß in wurzelunterschiedliche Personennamen notwendig ist. Die Antwort wäre einfach, es geht um das Aufhellen der geschichtlichen Verhältnisse und der auf den 
ersten Blick nicht wichtigen Zusammenhänge sowie um die Bedeutung der Arbeit der Namenforscher mit den handschriftlichen Quellen und um ihre präzise Auswertung. Das Anliegen der Autorin des Beitrages ist $\mathrm{zu}$ zeigen, dass heute fern $\mathrm{zu}$ scheinende Jahrhunderte und in ihnen liegende onomastische Zeugnisse nie ihren Wert sowie für die Namenforschung als auch für die Geschichte, für die Siedlungsgeschichte und die Erschließung der Sprach- sowie Völkerkontakte verlieren werden. $\mathrm{Zu}$ dem PN Tridden vgl. lit. trìdas 'schnell aufbrausend, eilfertig, hastig, Heißsporn', den lit. Übernamen Trìdas (Butkus 1995: 425). Die Entstehung der lit. Familiennamen war ein kompliziertes Prozess im onymischen System der lit. PN wie eigentlich auch in onymischen Systemen anderer Völker. In der 2. Hälfte des 14. Jhs. kann man kaum mit den lit. Familiennamen rechnen. Sie sind später entstanden (s. Maciejauskiené 1991: 50-54; 2007: 477-479). In dem angeführten Privileg geht es um die Einnamigkeit der Person. Der Name ist litauischer Herkunft und könnte als Übername nach Merkmalen des Charakters betrachtet werden. Solche Fälle der Benennung der Litauer sind selten in den historischen Quellen, deshalb verdienen sie besondere Aufmerksamkeit der Namenforscher sowie der Historiker, weil sie, in unserem Fall der angeführte PN, nicht zu der Schicht der ältesten zweistämmigen lit. Personenamen gehören. Vgl. die lit. hist. PN 1651 Urbanum Tridonis, Bräutigam, 1663 Valentino Tridonaitis, Trauzeuge; 1684 Anna Trydonaycia, Braut (Rayon Vilkaviškis, Gemeinde Lankeliškiai, unweit der Grenze zu Kleinlitauen/Preußisch-Litauen) (IAK). Im Ganzen lassen die besprochenen PN und ihre Verbreitung im Grenzbereich die Schlussfolgerung zu, dass der Heimatort des im Westsamland in Rogehnen begünstigten Litauers Tridden eben bei Tilsit gelegen hat.

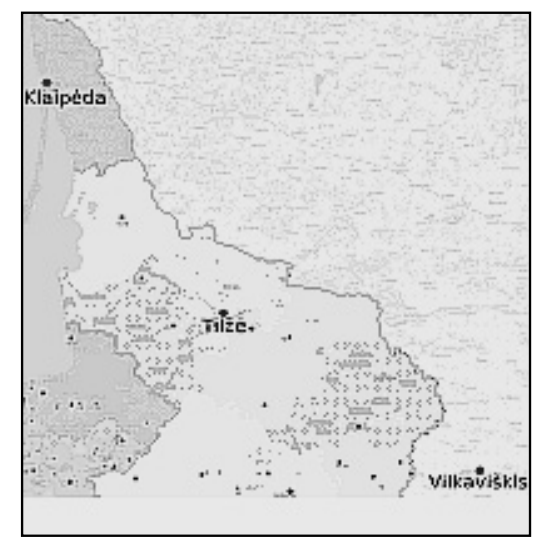

Auszug aus der Karte mit Tilsit, Klaipėda, Vilkaviškis und mit möglichen Wohnorten einiger erwähnten Personen (angefertigt von Vytautas Sveikauskas) 
1372 [Überschrift der Urkunde] Ibidem Vmbo

Wir Bruder winr[rich] von knypr[ode] homeist[er] de $\beta$ orden $\beta$ der brudere Deß spitaT Jencte marien de $\beta$ dut $\int$ chen huß von Jer[ufalem] - mit Rate vnfer mitgebiteg[er] • vorlyen vnd geben jutten vnd vmbo $\int y n e ß$ bruder $\int$ on den littowen vnd Iren recht[en] erben vnd nochk[omelingen] iiij hok[en] gelege uff dem velde c3u Rogyn Bynne den grenic5्रe al $\beta$ y In do von vn $\int[e r] n$ brud[er]n fynt bewifet ffry von c3्रenden vnd geburlichir erbeit mit recht[en] von..

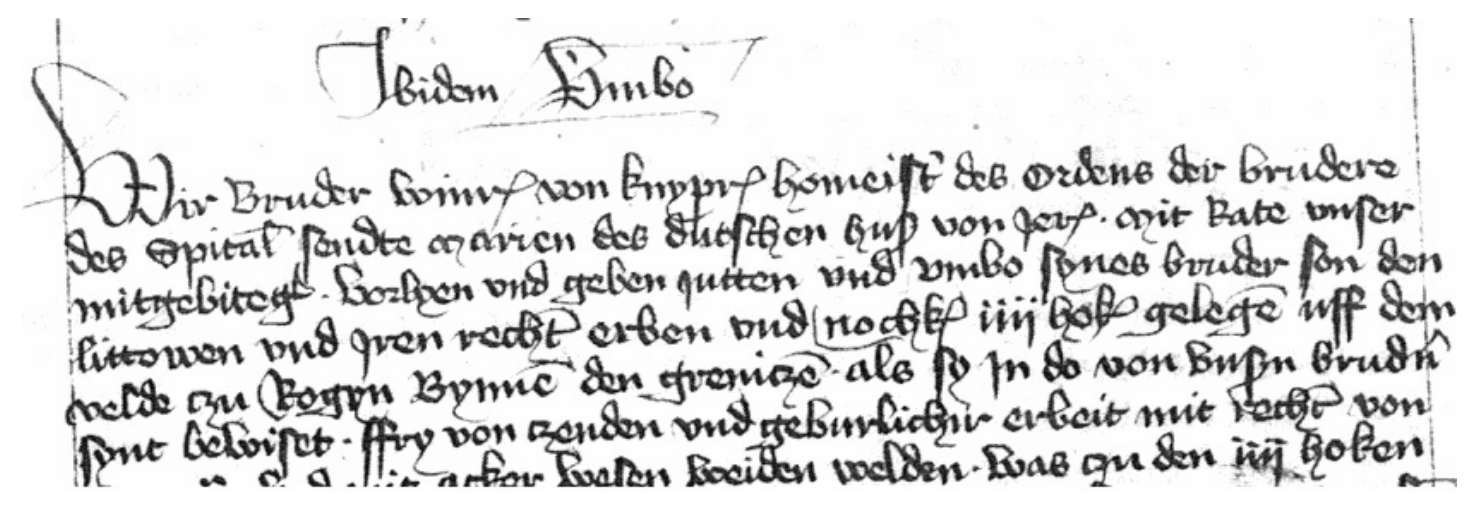

GStA PK, XX, HA, OF, Nr. 107, Bl. 128v

In Rogyn (Rogehnen) wurden 1372 noch zwei Litauer Jutte und sein Neffe Vmbo mit 4 Haken Land begünstigt. In der Kartei der litauischen historischen Personennamen sind einige Jut-Namen zu finden, wie z.B. 1554 Thomasz Juteykowicz im Vilniusser Gebiet (IAK). In LPDB werden einige PN wie Jutà, Jùtas, Jùtè, Juteikà, Jutèlis, Jùtis u.a. angegeben. Als Ausgangspunkt für die Erklärung dieser Namen gelten die poln. PN wie Juta, vgl. den poln. hist. PN 1663 Juta (AP II 122), auch die dt. PN Jutta, Jutt (Gottschald 1954: 356), vgl. auch den dt. Rufnamen Jutta von Judith (GVL 238). Zigmas Zinkevičius (2008: 598) betrachtet diese PN als lit. Übernamen, die die geistlichen Eigenschaften des Menschen offenbaren, und stellt sie zu lit. jut-ét 'wachen; fühlen' s. LKŽe. Um falsche Schlussfolgerungen zu vermeiden, ist folgendes hinzuzufügen, nicht alle in den Handschriften des Deutschen Ordens als Litauer definierten Personen trugen lit. PN. Das sollte mehr als verständlich sein. Aber in unserem Fall kann man mit dem muttersprachlichen appellativischen Nachweis dieses Personenamens rechnen, vgl. alit. jutùs 'wachsam, munter', schon von Zinkevičius angeführtes jutéti (jùtaljuti, jutéjo) (ALEW I 424-425) und lit. jutà 'Empfindung; Empfindlichkeit' (LKŽe). Es ist offensichtlich, dass man bei der Besprechung der ältesten Schicht der lit. PN alle Möglichkeiten ihrer Deutung als auch den historischen Hintergrund, in erster Linie Sprachkontakte, in Erwägung ziehen soll und dabei die Rolle der Muttersprache bei der Benennung der Personen nicht vergessen soll. 
Im Fall von Jutten kann man am ehesten für die lit. Herkunft des Personennamens plädieren. Der Name des Neffen von Jutten Vmbo kann vorläufig nicht gedeutet werden. Seine Ableitungsbasis ist im Litauischen nicht als Appellativum belegt. Es gibt keine Parallelen im anthroponymischen System der baltischen Sprachen sowie in den Systemen der möglichen Kontaktsprachen. Es bleibt zu warten bis der Name sprachlich gedeutet wird. Wichtige historische Informationen zur Erforschung der fremden Einflüssen, die das historische litauische Namengut liefert, dürfen nicht unberücksichtigt gelassen werden. Die beiden lit. PN Jutte und Vmbo sind nicht leicht durchschaubar, besonders der letzte. Ob man sie aufgrund ihrer Herkunft als Unika bezeichnen darf, ist fraglich.

Es liegt kein Grund zum Zweifel, dass die beiden Personen aus Litauen kommen, weil in der Handfeste auch auf das ap di land c3्र $u$ littowen ap Siland an Ruttionuc hingewiesen wird.

Das Dorf Rogin (Rogehnen) wurde im OF 107 1372-1400 (mit Registereinträgen) 6 Male erwähnt (man darf das spätere Rogehnen, Kreis Fischhausen im Kirchspiel Wargen nicht mit Rogahnen, Kreis Königsberg im Kirchspiel Heiligenwalde verwechseln, wie das bei Vercamer (2010: 421,439, 448) gemacht wird). Von 1400 bis um 1540 war Rogehnen zum adligen Sitz geworden (Vercamer 2010: 239). Diese Behauptung bestätigen die späteren Quellen: 1785 Rogehnen oder Rogen Adel. Gut mit 9 Feuerstellen, eingepfarrt zur Kirche Wargen (Goldbeck VT 153), 1844 Rogehnen ad. Gut mit 9 Wohnhäusern und 152 Einwohnern (Gebauer 152). Der Besitz im Dorfe betrug um die Jahre 1372-1400 512,4 Hektar Land, die ansässigen Litauer wurden davon mit 89,6 Hektar Land begünstigt.

Viele interessante Erkenntnisse verspricht auch der folgende Auszug einer Handfeste aus demselben Jahr 1372 (bei Vercamer (2010: 495) wird das Jahr 1394 angegeben; s. noch Blažienè 2000: 144-145):

1372 Auszug aus der Handfeste über die Verleihung des Landes an den

...vnd geben meifen dem lit//

Litauern Meifen

towen von poffeln vnd synen rechten erbn vnd nachk[omelingen] |iiij ho/ ck[en] gelegen vff dem velde c33u Surfen...

GStA PK, XX, HA, OF, Nr. 107, Bl. 125v-126r

Die Handfeste bietet zusätzlich die Annahme, dass im KA Wargen zwei Litauer aus derselben Gegend, aus dem Dorf Posseln (s. oben Passiln) ansässig waren. Die Wiedergabe des Siedlungs- 
namens unterscheidet sich durch zwei Buchstaben, aber man kann mit Sicherheit behaupten, dass es um einen und denselben Dorfnamen geht, und vorläufig ist m.E. zusätzliche Untersuchung überflüssig. Der beliehene Meifen bekam 4 Haken Land diesmal in Surfen, späteres Schorschehnen, jetzt Malinovka (s. Blažienè 2000: 144-145). Schorschehnen war nur 2,0 km von Rogehnen entfernt (OV 448) in dem sich der Litauer Tridden niedergelassen hat. Diese Tatsache veranlasst die Vermutung, dass zwei litauische Familien aus demselben Dorf bei Tilsit im samländischen Kirchspiel Wargen in unmittelbaren Nachbarorten ihre Unterkunft gefunden haben. Man könnte sie als Umsiedler, deren Namen höchstwahrscheinlich große Nähe zu den sprechsprachlichen Formen belegen, d.h. dass sie dem fremden Ohr der Ordensschreiber verständlich waren, bezeichnen. Zeugen diese Tatsachen von der Ausweitung des Siedelgebietes der Litauer nach Westen des Samlandes? Diese Frage bedarf sowie siedlungsgeschichtlich als auch bevölkerungsgeschichtlich sehr fundierter Antwort, die immer prüfenswert sein wird, weil die Siedlungspolitik des Deutschen Ordens und die relativ frühe den Prußen fremde Namenüberlieferung, gestützt auf die im Laufe der Zeit zuverlässiger bearbeiteten archivalischen Quellen, immer wieder von neuem Blickwinkel zu betrachten sind. Ohne möglichst genaue Dokumentation sind neue Erkenntnisse nicht vorstellbar.

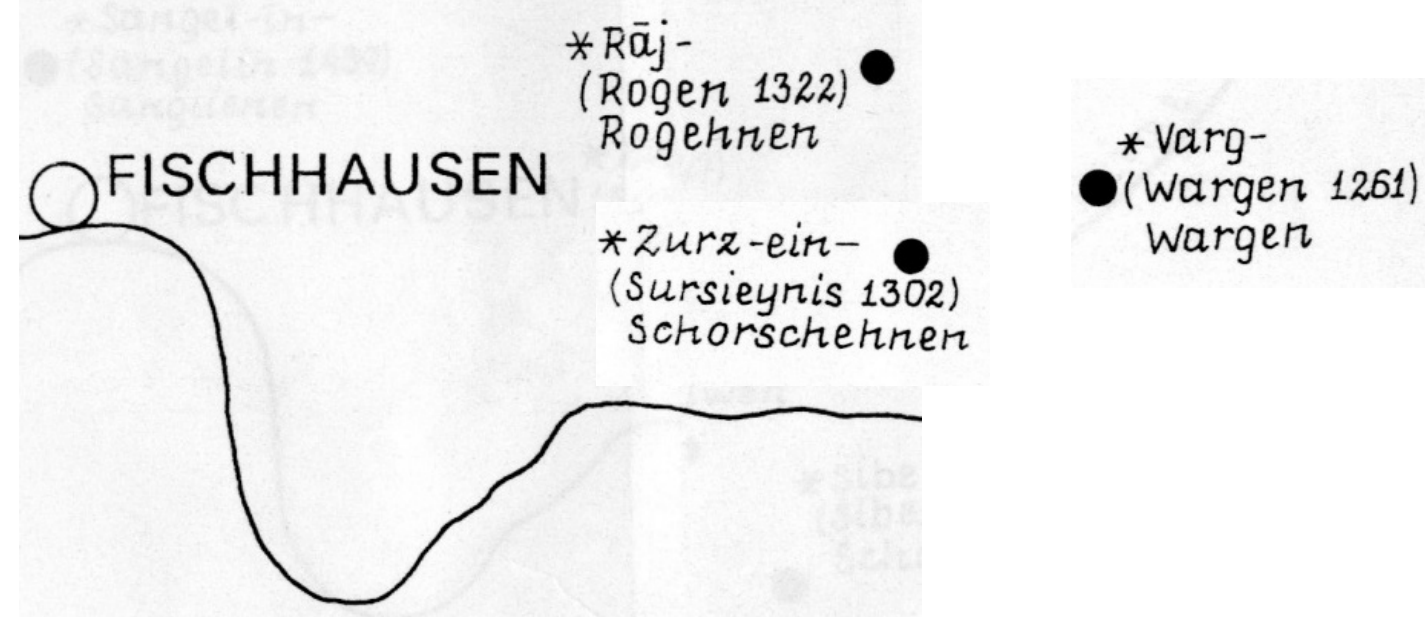

Auszug aus der Karte von Grasilda Blažienė mit den Ortsnamen Rogehnen und Schorschehen im KA Wargen.

$\mathrm{Zu}$ dem lit. PN Meisen vgl. die lit. PN 1545 Michal Meysos (Kaunas), 1664 Simon Meisinikaitis (Rayon Vilkaviškis, Gemeinde Lankeliškiai), 1691-1781 Joannes Meysiunas (Rayon Molètai, Gemeinde Videniškiai) (IAK), vgl. die lit. Appellativa meisà 'Fleisch', 
meĩsininkas 'Fleischer', verbreitet in Žemaitija (Niederlitauen) (LKŽe). Des Weiteren vgl. wegen der möglichen Problematik der Namenübertragung mit ei durch ai vielleicht die lit. PN 1539/1540 Maisotte im Amt Saalau (Ostpr. Fol. 911 a VIII 4 17v); 1540 Petrick Maysytter im Amt Memel (Diehlmann 2006: 22); 1659 Petro Maysim, Taufpate; 1680 Christophus Maysian, Taufpate aus Adutiškis; 1749 Mathias Majs, Trauzeuge aus Gruzdžiai (IAK). Zu mais- vgl. (?) lit. maĩse (lett. maize) 'Brot', in Klaipèda (Memel) hat das Appellativum die Bedeutung von 'Mehlsuppe' (LKŽe). Man hat in den meisten Fällen keinen anderen Weg zur Erörtung der PN, als nach der deappellativischen Herkunft zu suchen. Im Nachbardorf Surfen, späteres Schorschehnen, wurden zwei litauische Familien mit 4 Haken Land beliehen. Die Verleihung erfolgte unter denselben Bedingungen, wie sie schon von der Autorin beschrieben wurden bei der Behandlung der russischen Personennamen in den Dokumenten des Deutschen Ordens (s. Blažienè 2018: 109-124, hier 117).

1372 [Überschrift der Urkunde] Ibidem Legeyko

Wir Bruder Winrich von Knyprode homeist[er] deß orden $\beta$ der brudere de $\beta$ SpitaT $\int e n c t e$ marien de $\beta$ dut $\int s c h n$ huse $\beta$ von I[esrusalem]

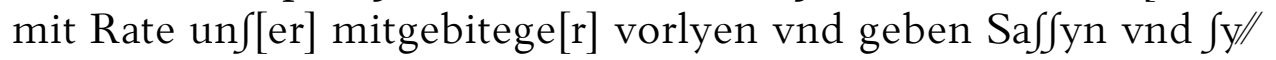
nen bruden den littouwen vnd iren rechten erbenn vnd nachk[omelingen] vier hock[en] gelegen vff dem velde c3u Surfyn... ouch vorlyen en von funderlichen gnaden ab di land c33u pafsiln do $\int y$

GStA PK, XX, HA, OF, Nr. 107, B1. 125r-126v

gewonet haben.

$\mathrm{Zu}$ dem PN Saffyn ist vorläufig nicht einfach etwas genaueres zu sagen. Man könnte sich an Reinhold Trautmann (1925: 90) schließen und den pruß. PN 1347 Sassin als Stütze für die mögliche Deutung nehmen. Trautmann führt noch denselben lit. PN Sassin an und vergleicht fragend die PN mit dem lit. ON Sasniten. Der PN Sasinas ist in Niederlitauen (Žemaitija) bezeugt und wird zu den slaw. PN wie der poln. PN Sasin gestellt (LPDB). Vgl. den poln. hist. PN 1578 Sasin (Sasinus) (AP IV 304). Wenn man sich auf die pruß. Angaben stützt, vgl. die pruß. ON Sasnyten, Sasnithen, die von der Autorin (2005: 176, 326) besprochen und auf das pruß. Appellativum sasnis, vgl. sasins 'Hase' (s. Mažiulis 2013: 818f.), zurückgeführt wurden. Wegen des Mangels an zuverlässigen onymischen und appellativischen Parallelen ist problematisch, Stellung zu der Deutung dieses lit. Personennamens zu nehmen. Im KA Wargen sind Litauer auch in den handschriftlichen Dokumenten 
der 1. Hälfte des 16. Jhs. notiert : 1540 die ander einwonner vnnd dorfschaffter des camerampts Wargenn Peter Littaw vorm hause (Ostpr. Fol. 911 a XXX 1 55v). Dieser Auszug stammt aus dem Türkensteuerregister von 1540 des Herzogtums Preußen. Dieses Register, das 36 Bände des sogenannten Ostpreussischen Folianten 911 a umfasst, entstand im angesicht der Türkenkriegsgefahr, um eine Türkesteuer im Herzogtum $\mathrm{zu}$ erheben, und enthält eine ausführliche Bevölkerungsliste mit sehr genauen Angaben über wirtschaftliche Verhältnisse. Siedlungsgeschichtlich, bevölkerungsgeschichtlich und von der Sicht der Eigennamenforschung ist die Quelle m.E. unentbehrlich und ist, leider, von den Namenforschern nicht ausgeschöpft am ehesten wegen dem Mangel paläographischer Kentnisse. Die Erschließung der frühneuzeitlichen Bestände der Archivalien braucht besondere Erfahrung (vgl die Ausgaben von dem größten Kenner dieser Bestände Hans Heinz Diehlmann der Jahre 1998, 2006, 2008). Die Autorin des Beitrages hat mit den Bänden des Folianten gearbeitet, die das Samland betreffen, und den höchsten Grad der Schwierigkeit bei der Entzifferung der Eigennamen erlebt. Personenbeschreibung wie auch genaue Indentifizierung des Kontextes sind eng verbunden und bieten oft unerschöpfliche Möglichkeiten für das Gesamtbild der Verhältnisse im Untersuchungsgebiet (s. Naumann 2011: 91).

In diesem Auszug geht es um den Litauer Saffyn, der eben in Passiln gewohnt hat und mit 4 Haken Land in 1300 Sursieine (Erstbeleg) (Schorschehnen) beliehen wurde. Schorschehnen war 1785 ein kleines Dorf eingepfarrt zur Kirche Wargen und hatte 5 Feuerstellen (Goldbeck VT 170). 1844 hatte das kleine Dorf 6 Wohhäuser und 63 Einwohner (Gebauer 123). Zu dem ON s. Blažienè 2000: 144-145. Nach dem genauen Einblick in OF 107 ist es gelungen auch den PN 1400 Sorfyon der krezcemer zu entdecken. Der neu gefundene PN ermöglicht, die Deutung des Ortsnamens zu revidieren und ihn als deanthroponymischen ON zu betrachten. Der Siedlungsname wurde von der Autorin des Beitrages im Jahre 2000 anders gedeutet. Die Schlussfolgerung lässt keinen Zweifel zu: die handschriftlichen Dokumente müssen mit akribischer Genauigkeit bearbeitet werden (vgl. Hengst 1994: 93-96).

Mit dem OF 107 aus handschriftlichen Beständen des Historischen Staatsarchivs zu Königsberg, verwahrt im Geheimen Staatsarchiv «Preußischer Kulturbesitz» in Berlin, wurde viel gearbeitet und, leider, einiges übersehen. Seit 2001 befasst sich die Autorin (Blažienè 2001: 16-17) mit den prußischen Appellativa, die von anderen Namenforschern als Eigennamen erforscht waren (s. noch 
Blažienè 2015: 26-31). Völlig zufällig wurde noch ein umstrittenes Appellativum, das nur als Ortsname bekannt war, entdeckt, der im Aufsatz näher erörtert wird.

\section{8}

\section{Ouch Rauketen briff}

Wir Bruder Johan von lorich voyt c3u Samland haben bewi Set Claws von poffindern vnd Rawketen vnd Sangeden $\int y n e n$ bruder vnd Iren rechten erben vnd nochkomelingen $\mathrm{E}$ eine halbe hube dameraw von deß obirsten marfchalk wegen der c33u mole marكchalk waß Bruder Rutl Rutcher von Elner by dem Selben dorffe gelegen al $\beta$ y Im bewifet ift c3u $c 3$ u Irem alden gute vff daß daß $\int \mathrm{y} v \mathrm{v} \beta$ defte baß gedyòe mo

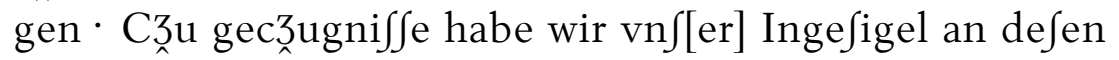
briff gehangen der gegeben ift In der Jarc3al vn $\int[\mathrm{er}] ß \mathrm{~h}[\mathrm{e}] \mathrm{rn} \mathrm{m}^{\circ}$ $\operatorname{ccc}^{\circ}$ lxxviij c3u Cremiten vff $\int e n t e$ Jurgen tag de $\beta$ fynt ge c33uge vnf[er] liben brude[re]·Bruderditerich von laran hufk[omptur] c3्र́u Konig[sberg] Bruder Burchart mauffeld vnf[er] Compa vnd vil andir erbar lute

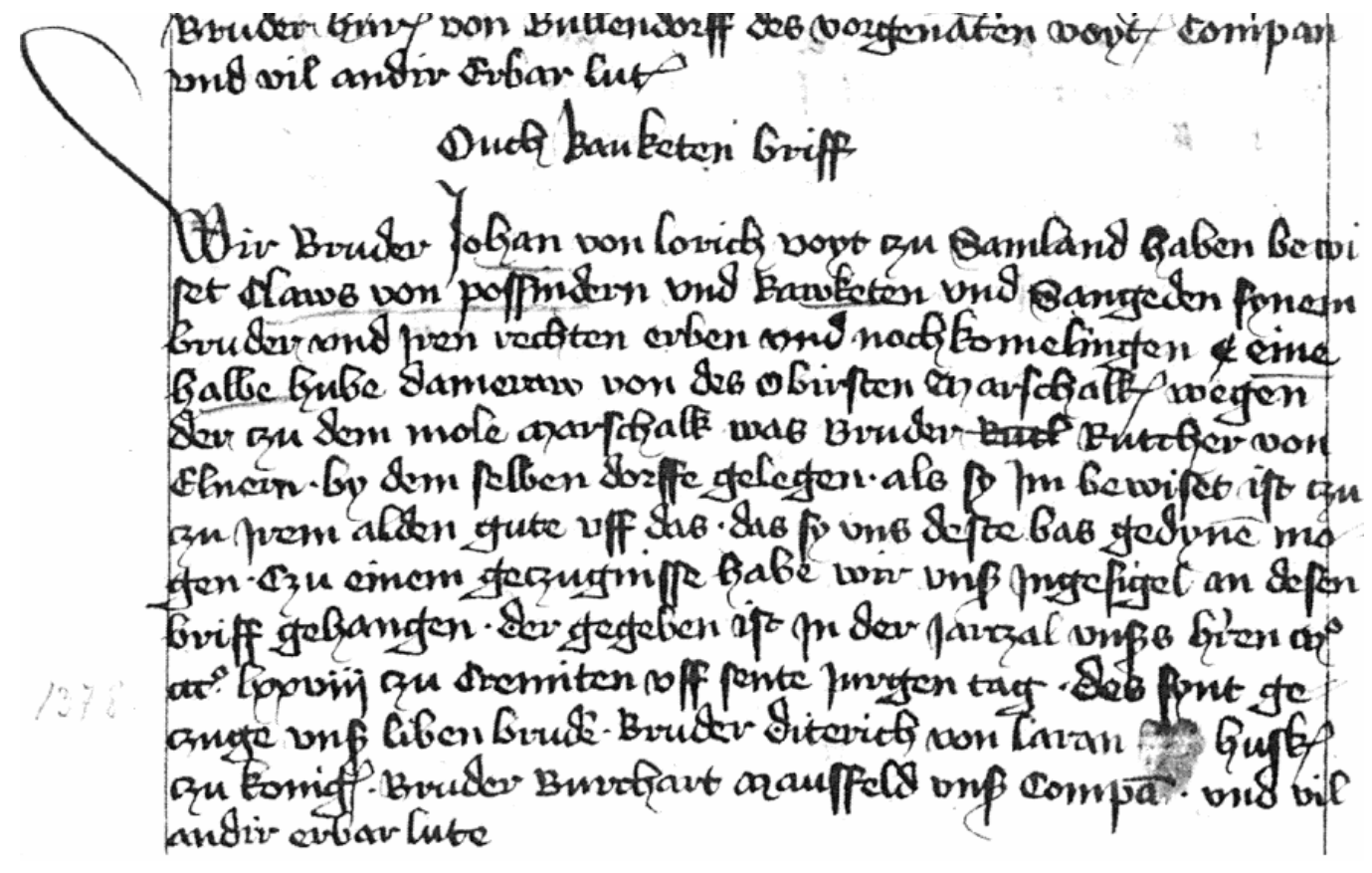

GStA PK, XX, HA, OF, Nr. 107, B1. 67v

In dieser Handfeste wird den Einwohnern von Possindern (späteres Possindern, jetzt Roščino) im Kr. Königsberg, Kirchspiel Heiligenwalde Claws und seinen Brüdern Rawketen und Sangeden eine halbe hube (nach heutigem Maß 8,4 Hektar) dameraw verschrieben. Leider wurden von der Autorin keine intensiveren Forchungen betrieben, als der Dorfname Damerau im Samland, von lautem Eifer möglichst mehr Neufunde zu präsentieren, fünfmal 
vorgestellt wurde (Blažienė 2000: 26). Dabei wurde nicht beachtet, dass dieser ON im südlichen Teil Ostpreußens (s. Prybytek 1993) und im anderen Teil von Ostpreußen (s. Blažienè 2005) nicht belegt war. Georg Gerullis (1922: 25) war zurückhaltender und hat nur zwei Eigennamen Dambraw, See und Dampraw, Dompraw angeführt, sie zu poln. dąbrowa 'Eichenwald' gestellt und mit der Bemerkung versehen, dass die Namen überall im Preußenlande zu finden sind. Vladimir Toporov (1975: 296) hat auch nur von dambrNamen gesprochen. Außer Acht wurde die Tatsache gelassen, dass im Elbinger Vokabular unter der Nummer 588 dameraw als das deutsche Wort für pruß. wangus steht (Mažiulis 1966: 71; 2013: 932). Die Bedeutung von wangus 'eine mit jungen Eichen bestandene, halb ausgerodete Waldfläche' war seit langem bekannt. 1595 beschreibt Caspar Hennenberger Damerawen (S. 8). 1848 widmet sich den altpreußischen Ortsnamen F. Neumann, der eben den Ortsnamen Damerau und die damit verwandten bespricht. Im Kommentar zu dem Aufsatz von Neumann betont Georg Heinrich Ferdinand Nesselmann, dass Damerau ein preußisches Wort zugrunde liege, welches etymologisch verwandt und ähnlich klingend dem polnischen Dabrowa gewesen sei (1848: 250). Dieselbe Meinung vertritt Nesselman auch 1873: 26-27, als er in seinem Thesaurus damerau bespricht und das Wort aus den Urkunden des Monumenta Historiae Warmiensis anführt. Nesselmann hat kein Beispiel aus den das Samland betreffenden Urkunden vorgelegt. Wie aus der Urkunde ersichtlich wird, kommt in ihr das Appellativum damerau vor, das wohl eine Heidefläche mit unausgewachsenen Eichen bedeuten konnte und die den Beliehenen irgendwelche Vorteile darbot. Genaue Hinweise auf die Bedeutung von damerau in diesem konkreten Fall bedürfen noch sehr genauen Erläuterungen. Der Hinweis auf diese Urkunde und das mögliche von anderen Forschern noch nicht erfasstes Appellativum sollte den Baltologen und Slawisten seine Bedeutung anschaulich machen, damit sie der Aussagefähigkeit von damerau wesentliche Aufmerksamkeit schenken könnten. Der Siedlungsname Possindern, der zu den schwer erklärbaren Namen gehört, zeichnet sich durch eine der einheitlichsten Belegreihen im prußischen Namengut aus (s. Blažienè 2000: 121).

In dem untersuchten Foliant wurde eine reizende Zeichnung entdeckt, die den Lesern auch zur Verfügung gestellt wird. Solche vielsagenden Zeichnungen kommen in den von mir bearbeiteten Folianten des Deutschen Ordens nicht oft vor, offen gesagt das war auch ein Neufund. 


\section{4}

In no[min]e d[omin]i amen Omnib[us] Chri[sti] fidelib[us] p[rese]nt[is] $1[$ itte] $\overline{r e}$ tenorem intuentibus eui dentius innote $\int$ cat Noß fr[ater] Theoder[icus] de Aldenburg ${ }^{\circ}$. ordiniß fr[atru]m dom[us] teu[tonicorum] marsch[alkus] Ex $\int$ citu et voluntate [ac] con $\int[$ en] ßu maturo venéabiliß viri fr[atris] luderi de Brunfwig elufdem ordiniß m[a]g[ist]ri generaliß C Contulim[us] Billote duoß uncoß In campo wergenow fitoß eo Iure habendoß retine $\lceil n\rceil d o s . .$.

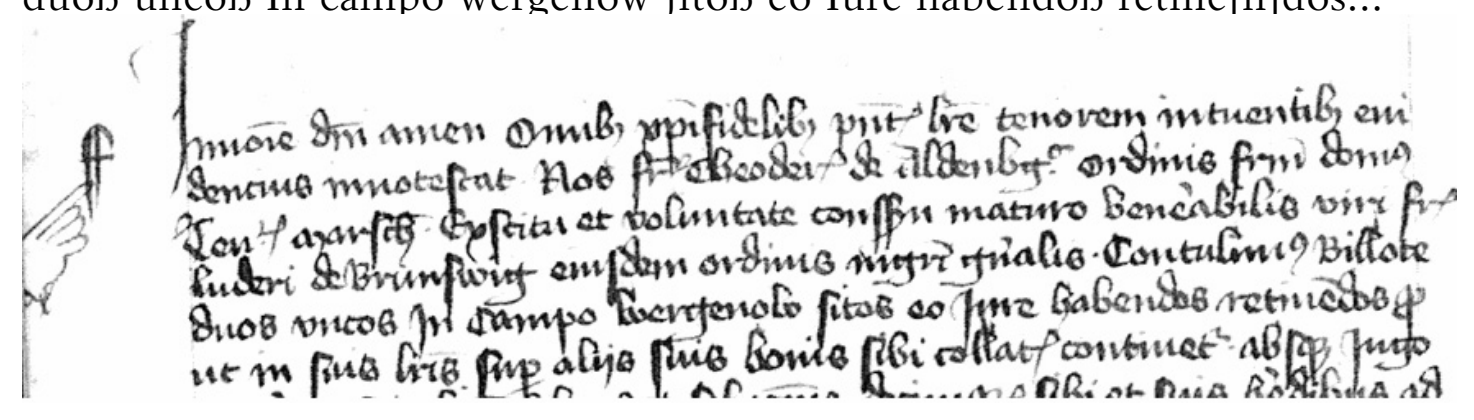

In der auf Latein geschriebenen Urkunde geht es um die Verleihung von zwei Haken Land an Billote in Wergenow, Erstbeleg im Jahre 1255 (späteres Wargenau) im Kr. Fischhausen, Kirchspiel Rudau. Der Zeigefinger ist auf die Anfangsworte «In nomine domini amen» (Im Gottes Namen amen) gerichtet. Mit diesen Worten ist am ehesten und das gezeichnte Kreuz verbunden. Der Schreiber der Urkunde ist unbekannt. Die Urkunde stammt nicht von der Hand Peter von Wormditt (zu ihm s. Blažienè 2018: 110). Diese Überlegungen tragen den hypothetischen Charakter.

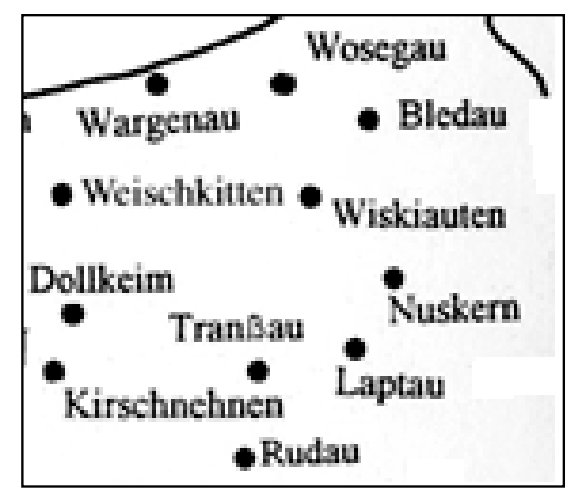

Wargenau im KA Rudau aus der Karte von Grasilda Blažienė

Wie das angeführte Material als auch die Überlegungen der Autorin zeigen, dass man eigentlich größeres Vertrauen der ziemlich exakt wirkenden Ordenskanzlei und ihren Schreibern schenken sollte. Man darf nicht auf historische Belege und Vergleichsnamen verzichten, um die Eigennamen, in unseren Fall die Personennamen, zu deuten. Ideal wäre die Handschriften mit dem Namengut für die 
Namenforschung und zugleich für die verstorbene Sprache der alten Prußen mehrfach $\mathrm{zu}$ behandeln und $\mathrm{zu}$ edieren. Das vorhandene Material soll auf alle Fälle nutzbar gemacht werden, deshalb träumen wir von den Namenbüchern mit historischen Überlieferungen. Auf das Anführen von Belegen unf Vergleichsnamen darf nicht verzichtet werden.

\section{Literatur}

ALEW - Altlitauisches etymologisches Wörterbuch. Unter der Leitung von W. Hock und der Mitarbeit von E.-J. Bukevičiūtè und Ch. Schiller, bearbeitet von R. Fecht, A. H. Feulner, E. Hill und D. S. Wodko. Bd. 1-3. Hamburg: baar, 2015.

AP II - [Autorenkollektiv] Antroponimia Polski od XVI do kónca XVIII wieku. HA - Ma. Pod redakciją A. Cieślikowej. Kraków: Wydawnictwo LEXIS, 2009.

AP IV - [Autorenkollektiv] Antroponimia Polski od XVI do kónca XVIII wieku. $M l-S t$. Pod redakciją A. Cieślikowej. Kraków: Instytut Języka Polskiego PAN, 2013.

ATSŽ - Lietuvos TSR administracinio-teritorinio suskirstymo žinynas. Vilnius: Mintis, 1976.

Blažienè, G. 2000. Die baltischen Ortsnamen im Samland (=Hydronymia Europaea, Sonderband II). Stuttgart: Franz Steiner Verlag.

Blažienè, G. 2001: Zu den altpreussischen Ortsnamen im Samland. In: Tarptautine Kazimiero Bügos konferencija: Etimologija ir onomastika. Vilnius, $2001 \mathrm{~m}$. lapkričio 9 d. Konferencijos tezès. Vilnius: VU Baltistikos ir bendrosios kalbotyros katedra, 14-17.

Blažienė, G. 2005: Baltische Ortsnamen in Ostpreußen (=Hydronymia Europaea, Sonderband III), Stuttgart: Franz Steiner Verlag;

Blažienè, G. 2015: Kalbos lemtis ir likimas. In: Nerimas 3. Vilnius: Lietuvos mokslu akademija, 16-49.

Blažienè, G. 2018:. Zu den russischen Personennamen in den Folianten des Deutschen Ordens. In: Indoevropeiskoe yazykoznanie i klassicheskaya filologiya [Indo-European linguistics and classical philology] 22 (1), 109-75. Zutritt : https://tronsky.iling.spb.ru/static/tronsky 2018_01.pdf. ISSN 2306-9015, DOI:10.30842/ielcp230690152209.

Būga, K. 1911/1958: Lietuvos kunigaikščiu vardai. In: Rinktiniai Raštai I. Vilnius: Valstybinè politinès ir mokslinès literatūros leidykla, 232-269.

Butkus, A. 1995: Lietuviu pravardès. Kaunas: ÆSTI.

Diehlmann, H. H. 1998: Die Türkensteuer im Herzogtum Preußen 1540. Band 1. Fischhausen- Schaaken-Neuhausen-Labiau. Hamburg: Verein für Familienforschung in Ost- und Westpreußen.

Diehlmann, H. H. 2006: Die Türkensteuer im Herzogtum Preußen 1540. Band 2. Memel-Tilsit. Hamburg: Verein für Familienforschung in Ost- und Westpreußen.

Diehlmann, H. H. 2008: Die Türkensteuer im Herzogtum Preußen 1540. Band 3. Ragnit-Insterburg - Georgenburg-Saalau. Hamburg: Verein für Familienforschung in Ost- und Westpreußen. 
Gebauer, K. E. 1844: Kunde des Samlandes oder Geschichte und topographisch-statistisches Bild der ostpreussischen Landschaft Samland. Königsberg: Universitäts-Buchhandlung.

Gerullis, G. 1922: Die altpreußischen Ortsnamen. Berlin und Leipzig: Walter de Gruyter \& Co.

Goldbeck, J. Fr. 1785: Vollständige Topographie des Königreiches Preußen. Bd. 1. Königsberg; Leipzig: Kanter.

Gottschald, M. 1954: Deutsche Namenkunde. Unsere Familiennamen nach ihrer Entstehung und Bedeutung. Berlin: de Gruyter.

Gudavičius, E., Matulevičius, A. 2013: Traidẽnis.In: Visuotine lietuviu enciklopedija XXIV Tolj-Veni. Vilnius: Mokslo ir enciklopedijų leidybos centras, 64.

GVL - Duden. Das große Vornamenlexikon. 4. Völlig neu bearbeitete Auflage. Bearbeitet von Rosa und Volker Kohlheim. Dudenverlag: Berlin-Mannheim-Zürich, 2014.

Fraenkel, E. 1962 -1965: Litauisches etymologisches Wörterbuch. Bd. 1-2. Heidelberg: Winter.

Hengst, K. 1994: Urkunde und Ortsname. Zum Typ der Ortsnamen auf itzsch - Der Ortsname Culitsch -. In: Onomastica Slavogermanica XXI, 93-96.

Hennenberger, C. 1595: Erclerung der preüssischen grösseren Landtaffel oder Mappen, Königsberg: Georg Osterberger.

IAK - Abécèlinè istoriniu asmenvardžiu kartoteka, esanti Lietuvių kalbos instituto Baltų kalbu ir vardyno tyrimu centre. (Die alphabetische Kartei der historischen Personennamen, die im Zentrum für Forschung der baltischen Sprachen und des Namengutes des Institutes für litauische Sprache aufbewahrt wird).

LKŽe - Lietuvių kalbos žodynas 1-20, 1941-2002, elektroninis variantas, redaktorių kolegija: Gertrūda Naktinienè (vyr. red.), Jonas Paulauskas, Ritute Petrokienè, Vytautas Vitkauskas, Jolanta Zabarskaitè. Vilnius: Lietuvių kalbos institutas, 2005 (atnaujinta versiia, 2008). Zutritt: <http://www.lkz.lt/>

LPDB - Lietuviu pavardžiu duomenu bazè. Zutritt http://lkiis.lki.lt

LVKŽ - Kuzavinis, K., Savukynas, B. Lietuviu vardu kilmès žodynas. Vilnius: Mokslo ir enciklopedijų leidybos institutas, 2007.

Maciejauskienè, V.1991: Lietuviu pavardžiu susidarymas XIII-XVIII a. Vilnius: Mokslas.

Maciejauskienè, V. 2007: Das litauische Personennamensystem. In: Europäische Personennamensysteme. Ein Handbuch von Abasisch bis Zentralladinisch. Anläßlich der 65. Geburtstage von Rosa Kohlheim und Volker Kohlheim. Hrsg. von Andrea Brendler und Silvio Brendler. Mit einem Geleitwort von Ernst Eichler. Hamburg: Baar, 474-484

Mažiulis, V. 1966: Prūsu kalbos paminklai. T. I. Vilnius: Mintis.

Mažiulis, V. 2013: Prūsų kalbos etimologijos žodynas. Vilnius: Mokslo ir enciklopediju leidykla.

Naumann, H. 1986/2011: Zur Untersuchung von Eigennamen unter funktional-kommunikativem Aspekt. In: Namen in Sprache und Gesellschaft. Theoretische und methodische Probleme der 
Onomastik. Hrsg. von Andrea Brendler und Silvio Brendler. Hamburg: Baar, 87-93.

Nesselmann, G. H. F. 1873: Thesaurus linguae Prussicae. Der preussische Vokabelvorrath soweit derselbe bis jetzt ermittelt worden ist nebst Zugabe einer Sammlung urkundlich beglaubigter Localnamen. Berlin: Fer. Dümmlers Verlag. Harwitz \& Grosmann.

Neumann, F. 1848. Über altpreussische Ortsnamen mit Nachträgen von Nesselmann G. H.F. In: Neue preussische Provinzialblätter V, 241$249 ; 249-251$.

OF - Ordensfolianten aus dem Geheimen Staatsarchiv «Preußischer Kulturbesitz».

Ostpr. Fol. - Ostpreussische Folianten aus dem Geheimen Staatsarchiv «Preußischer Kulturbesitz».

OV - Orts-Verzeichnis nebst Entfernungs-Tabelle der Provinz Ostpreussen. Bonn: Buch- und Kunst-Handlung, [1893].

Pèteraitis, V. 1997: Mažosios Lietuvos ir Tvankstos vietovardžiai. Vilnius: Mokslo ir enciklopedijų leidybos institutas.

Przybytek, R. 1993. Ortsnamen baltischer Herkunft im südlichen Teil Ostpreußens (=Hydronymia Europaea, Sonderband). Stuttgart: Franz Steiner Verlag.

Toporov, V. N. 1975: Prusskiy yazyk. Slovar' [The Prussian Language. Dictionary]. Vol. I. Moscow: Nauka.

Топоров, В. Н. 1975: Прусский язык. Словарь. Т. 1. М.: Наука.

Trautmann, R. 1925: Die altpreußischen Personennamen. Göttingen: Vandenhoeck \& Ruprecht.

Vercamer, G. 2010: Siedlungs-, Verwaltungs- und Sozialgeschichte der Komturei Königsberg im Deutschordensland Preußen (13.-16. Jahrhundert $][=$ Ein+zelschriften der Historischen Kommission für ost- und westpreußische Landesforschung, Bd. 29]. Marburg: fibre.

Wenzel, W. 2009: Die sorbisch-deutsche Siedlungsverhältnisse zwischen mittlerer Elbe und oberer Spree im Spiegel der Personennamen. In: Slawen Deutsche Namen. Beiträge zur westslawischen Personenund Ortsnamenforschung. Mit besonderer Berücksichtigung des Sorbischen. Hrsg. von Silvio Brendler und Karlheinz Hengst, Hamburg: baar, 67-71.

Zinkevičius, Z. 2008: Lietuviu asmenvardžiai. Vilnius: Lietuvių kalbos institutas. 